Review

\title{
Herpesvirus acts with the cytoskeleton and promotes cancer progression
}

\author{
Yingfen $\mathrm{Wu}^{1,2,3}$, Fang Wei², Le Tang2, Qianjin Liao',4, Hui Wang 4 , Lei Shi², Zhaojian Gong2, Wenling \\ Zhang', Ming Zhou1,2,3, Bo Xiang 1,2,3, Xu Wu1,2,3, Xiaoling Li1,2,3, Yong Li2,5, Guiyuan Li1,2,3, Wei Xiong ${ }^{1,2,3}$, \\ Zhaoyang Zeng1,2,3, Fang Xiong ${ }^{1,2}$, Can Guo ${ }^{1,2,3}$ \\ 1. NHC Key Laboratory of Carcinogenesis, Xiangya Hospital, Central South University, Changsha, Hunan, China \\ 2. The Key Laboratory of Carcinogenesis and Cancer Invasion of the Chinese Ministry of Education, Cancer Research Institute, Central South University, \\ Changsha, Hunan, China \\ 3. Hunan Key Laboratory of Nonresolving Inflammation and Cancer, Disease Genome Research Center, the Third Xiangya Hospital, Central South University, \\ Changsha, Hunan, China \\ 4. Hunan Key Laboratory of Translational Radiation Oncology, Hunan Cancer Hospital and the Affiliated Cancer Hospital of Xiangya School of Medicine, \\ Central South University, Changsha, Hunan, China. \\ 5. Department of Cancer Biology, Lerner Research Institute, Cleveland Clinic, Cleveland, Ohio, USA \\ $\square$ Corresponding authors: xiongf@csu.edu.cn; guocde@csu.edu.cn; Tel.: 86-731-8480-5446;
}

(c) Ivyspring International Publisher. This is an open access article distributed under the terms of the Creative Commons Attribution (CC BY-NC) license (https://creativecommons.org/licenses/by-nc/4.0/). See http://ivyspring.com/terms for full terms and conditions.

Received: 2018.09.26; Accepted: 2019.03.14; Published: 2019.05.21

\begin{abstract}
The cytoskeleton is a complex fibrous reticular structure composed of microfilaments, microtubules and intermediate filaments. These components coordinate morphology support and intracellular transport that is involved in a variety of cell activities, such as cell proliferation, migration and differentiation. In addition, the cytoskeleton also plays an important role in viral infection. During an infection by a Herpesvirus, the virus utilizes microfilaments to enter cells and travel to the nucleus by microtubules; the viral DNA replicates with the help of host microfilaments; and the virus particles start assembling with a capsid in the cytoplasm before egress. The cytoskeleton changes in cells infected with Herpesvirus are made to either counteract or obey the virus, thereby promote cell transforming into cancerous ones. This article aims to clarify the interaction between the virus and cytoskeleton components in the process of Herpesvirus infection and the molecular motor, cytoskeleton-associated proteins and drugs that play an important role in the process of a Herpesvirus infection and carcinogenesis process.
\end{abstract}

Key words: Herpesvirus; cytoskeleton; drugs; infection; cancer

\section{Introduction}

\section{Herpesvirus and its life cycle}

Herpesviruses are a family of enveloped double-stranded linear DNA viruses that contain subfamilies that are classified as alpha, beta, and gamma herpesviruses [1]. Members of the alpha herpesvirus subfamily include the human pathogens herpes simplex virus (HSV), and varicella-zoster virus $(\mathrm{VZV})$, as well as the animal pathogens pseudorabies virus (PRV), bovine herpesvirus, and Marek's disease virus, which proliferate rapidly and cause cytopathic effects [2]. The beta herpesvirus subfamily includes, human and mouse cytomegalovirus (HCMV and
MCMV, respectively), with a long growth cycle, and infection by these viruses enlarges cells [3]. The gamma herpesvirus subfamily contains the lymphotropic Epstein-Barr virus (EBV) and Kaposi's sarcoma-associated herpesvirus (KSHV), whose target cells are mainly lymphoid cells that cause lymphoproliferation [4] Human gamma herpesvirus is closely related to the occurrence of some malignant tumors, such as EBV associated with Burkitt's lymphoma, Hodgkin's disease, nasopharyngeal carcinoma (NPC) [5-15], HHV-8/KSHV and Kaposi's sarcoma (KS) (the most common tumor in AIDS patients) [16], primary effusion lymphoma(PEL) [17] 
and multicentric Castleman disease(MCD) [18]. The virions of herpesviruses are made of DNA genome and an outside protein aceous capsid. Several proteins surrounding the capsid are called the tegument. There are multiple viral glycoproteins surrounding the tegument in an envelope.

The life cycle from herpesvirus entering the host to egress is a complex process in terms of space and time. First, herpesviruses enter cells by fusion with the plasma membrane or are endocytosed to the cell to eventually fuse within an intracellular vesicle or clathrin endocytosis pathways [19]. During this process, viral glycoproteins ( $\mathrm{gB}, \mathrm{gH} / \mathrm{gL}, \mathrm{gD})$ and host cell surface receptors play key roles [20].However, except HSV, HSV is preferentially taken up into glioma cells through an endosomal pathway rather than through fusion with the cell surface[21].The herpesvirus enters the cell and releases the viral capsid. Once the virus capsids enter the cytoplasm, the tegument proteins fall from the capsid, bind with the microfilaments and reach the centrioles and nuclei that are retrograding along the plus-end of the microtubules [22]. After the virus enters the nucleus, the virus begins DNA replication and macromolecular synthesis. When those actions are completed, the virus particles are packaged into mature viruses with capsids before release to the extracellular space. The virus life cycle in a host cell cannot proceed without the help of the intracellular cytoskeleton.

\section{Cytoskeleton components and related proteins}

The cytoskeleton is a complex intracellular network that consists of three components: microfilaments, microtubules and intermediate filaments [23-25]. Microfilaments are composed of actin filaments, and there are two forms of actin in vivo: G-actin and F-actin (i.e., the actin monomer and actin monomers assembled in fibrous actin). Actin has ATPase activity because of an ATP binding site in the inner side of the monomer. Actin monomers bind with ATP and initiate microfilaments assembly. Most of the microfilaments in the cell are concentrated in the cytoplasmic area and cross-linked with the microfilament-binding proteins to form a cell cortex involved in phagocytosis and other processes. Microfilaments also form stress fibers in focal adhesions, pseudopods and microvilli. The functioning of the microfilament network depends on a combination of microfilament-binding proteins, including fascin, cofilin and other proteins [26]. In addition, the molecular motor myosin, especially myosin $\mathrm{V}(\mathrm{MyoV})$ and MyoVI, specifically binds to the filaments and utilizes energy generated by the hydrolysis of ATP to transport cargo along microfilaments [27-29].

Other important components of the cytoskeleton are microtubules (MTs). Microtubules are hollow tubular structures composed of alpha- and beta-tubulin heterodimers. Alpha- and beta-tubulin heterodimers form fibrils through nucleation, and then those fibrils form microtubules by elongation in the microtubule organizing center (MTOC)[30]. Due to the different speeds of microtubules extension at both ends (the faster end called the plus end, the other is the minus end), thus making microtubules be in a polar state [31]. This polarity keeps microtubules in a dynamic state. The dynamics and distribution of microtubules are influenced by various factors, including GTP hydrolysis, posttranslational modifications of tubulin subunits, and microtubule-associated proteins [32]. Microtubuleassociated proteins (MAPs) are a class of proteins that are present along with the assembly of microtubules and are classified as MAP1, MAP2, MAP3, MAP4, tau, and others. Those proteins which have one or several microtubule-binding domains can stabilize microtubules as well as regulate microtubule network distribution and function. The targeted transports between microtubules to microtubules and membrane vesicles are regulated by two types of molecular motors: the plus end motor, kinesins, which is the transport toward the outer cell periphery. Kinesin consists of a motor domain in the shape of a sphere and a rod-shaped structure that contains ATP-binding sites and microtubule-binding sites [33]. The minus end steering motor, dynein, transports from the outer edge of the cell to the center. Dynein is the largest and fastest moving member of motor proteins [33-35].

The intermediate filament (IF) is also one of the components of the cytoskeleton. The IF is a rope-like structure with a highly conserved alpha spiral rod region in it. IFs are largely responsible for providing mechanical integrity for a cell, and they provide a scaffold for proteins involved in polarized trafficking [36]. Several well-known examples include keratins, neuro filaments, vimentin, desmins, and lamin A/C. In the process of virus infection, the role played by the intermediate filaments is less clear than other components of the cytoskeleton, in consist of impacts of virus infection.

The purpose of this paper is to elucidate the role of the cytoskeleton and associated proteins in the process of herpesvirus infection and the effects on the cytoskeleton after cell infection with the herpesvirus, thereby promoting tumor development [37-39]. 


\section{The Interaction between Herpesvirus and the Cytoskeleton during Virus Infection}

\section{Microfilaments Interact with Herpesvirus during Infection}

Once herpes virions make contact with cells, they encounter the cortical actin meshwork lining the cytosolic side of the plasma membrane, and the actin molecules connected to the cell surface receptors. Virion glycoproteins bind to specific host cell receptors during an infection (Figure 1). Ultimately, the envelope and plasma membrane fuses, delivering the capsid and tegument into the cytosol [40].

Viruses regulate the morphology and dynamics of microfilaments in cells by interacting with the surface receptors of cells. Binding of HSV-1 gD to the surface receptor nectin-1 stimulates Rho GTPase signaling or induces Cdc42 signaling [41-44], which in turn alter them or phology of cortical actin [45]. KSHV interacts with the cell surface receptors ephrin A2 receptor (EphA2R) and $\alpha 3 \beta 1, \alpha V \beta, \alpha V \beta 5$ integrins, which results in simultaneous induction of FAK, Src, PI3-K, Rho-GTPase signal and adaptor molecules that play critical roles in actin dynamics [46-49]. A dynamic actin cytoskeleton is necessary for viral entry, intracellular migration, and virion release [50-52]. When virus egress, the tegument protein VP22 of HSV-1 interacts with non-muscle myosin IIA (NMIIA), have implications for virions as they enter the extracellular environment [53-58]. Also, the KSHV $\mathrm{gB}$ mediates entry through association with the $\alpha 3 \beta 1$ integrin receptor and activates Rac1, Cdc42 and RhoA, then induces the formation of actin stress fibers and filopodia [59].

The impact on microfilaments not just happened on the surface, but also in the cytoplasm. Transport from the cell periphery to nucleusis mediated by microtubules and the molecular motor dynein. However, actin rearrangement and actin stress fibers are associated with viral spread. $\mathrm{U}_{\mathrm{S}} 3$, which is a conserved alpha herpesviral serine/threonine kinase, leads to PKA-dependent S188 RhoA phosphorylation, which is one of the hallmarks of suppressed RhoA signaling, Us 3 -mediated actin rearrangements, actin stress fiber breakdown and protrusion formation, as well as increased viral spread [60]. Actin stress fibers may also be affected by KSHV Kaposin B protein, and



Figure 1. Model of herpesvirus entry, DNA replication and egress with the help of microfilaments. A. The activity arises in cortex and cytoplasm when a herpesvirus enters and egresses. B. The activity occurs in the nuclear periphery and inside. 
KSHV Kaposin B protein can cause alterations in endothelial cell physiology, including the formation of long parallel actin stress fibers that depend on Kaposin B-mediated activation of a noncanonical signaling pathway that includes the stress-inducible kinase MK2, hsp27, p115 Rho GEF and Rho.

The activity of the virus in the nucleus is related to actin (Figure 1). The nuclear Herpesvirus capsid motility is active, directed, and uses nuclear filamentous actin to transport capsids through the nucleoplasm [27, 28]. And the movement of capsid along the actin filaments to perinuclear area may be driven (at least partly) by myosin [27, 28]. The appearance of nuclear actin filaments is associated with viral capsid assembly, and the appearance of nuclear actin filaments prior to assembly of the viral capsid is essential for the formation of viral capsid assembly sites. Transcription of the virus is also necessary for the formation of actin filaments [27]. Infection of neurons with PRV and HSV-1, which are alpha-herpesviruses, led to the formation of actin filaments in the nucleus, whereas uninfected nuclei did not contain actin filaments. The advances along actin filaments of PRV and HSV-1 viruses occurred in a myosin-dependent manner [27]. However, Jens B. Bosse found that herpesvirus nuclear capsid motility is not dependent on F-actin [61]. During infection with human cytomegalovirus (HCMV), viral DNA synthesis occurs at the periphery of replication compartments. Within the nuclear interior, and then, the assembled capsids must reach the inner nuclear membrane for translocation to the cytoplasm (nuclear egress). Actin filaments (F-actin) play an important role during the processes that facilitate movement of HCMV capsids to the inner nuclear membrane during nuclear egress. Actin is localizing along the RC periphery and between the RC periphery and the nuclear rim [62]. KSHV infection could increase both the transcription and protein levels of guanylate-binding protein 1 (GBP1) at the early stages of primary infection by activating the NF- $\mathrm{KB}$ pathway. However GBP1 significantly inhibited KSHV infection by inhibiting the nuclear delivery of KSHV virions through disrupting actin filament formation with its GTPase activity and dimerization [63].

\section{Interaction between Microtubules and Virus Infection}

Viruses must enter the nucleus when viruses need to copy and package their DNA. During adsorption into the nucleus, viruses avoid in the cytoplasm of viscous liquid and many organelles, as well as rely on the transport system of host cells, which is a time-saving method. Microtubules, which are one of the cytoskeletal components, play an important role in viral transport [64]. The MTOC assembled by microtubules is located beside the nucleus and is very convenient and effective for virus to enter the nucleus along microtubules. In addition, with the help of molecular motors, orientation driven transport through kinesins and dyneins, regulates the transport of microtubules, which makes MT the "highways" of microtubule transportation [65]. Viruses retrograde transport along microtubule minus ends towards microtubule-organizing center [66].

\section{The Interaction between Microtubules and Viruses in Cytoplasm}

Twenty-three capsid proteins on the surface of the virus are exposed to the cytoplasm when the virus enters the cell, or the virus is assembled. Endothelial capsid proteins such as proteins pUS3, pUL36, pUL37, ICP0, pUL14, pUL16, and pUL21 recruit dynein, dynactin, kinesin- 1 and kinesin-2. The capsid proteins which are covered by coat proteins including vhs, pUL11, ICP4, ICP34.5, VP11/12, VP13/14, VP16, VP22 and pUS11, then combine with microtubule motor molecules. Viruses bind to different molecules in the cytoplasm through their own proteins that carry viruses either to the nucleus or membrane [67]. The outer HSV capsid protein, VP26, interacts with the dynein light chains RP3 and Tctex1 [68].The HSV-1 UL34 protein (a primary envelope component) interacts with the dynein intermediate chain [69]. Some outer tegument proteins, such as VP13/14, VP16, and VP22, were lost during viral entry process and did not move with capsids to the nucleus [70]. But inner tegument proteins VP1/2 and UL37 still associate with capsids as they enter into neurons, subsequently, they transport into the nucleus and then dock at the nuclear rim [70]. And inner tegument proteins promote capsid transport along microtubules by interacting with the dynein/dynactin complex [71]. HSV inner tegument proteins UL36 (VP1/2) and UL37 make the movement of capsids more efficient in vitro [71]. The interaction of the UL36p (VP1/2) with the dynein/dynactin microtubule motor complex during retroviral herpes virus pseudorabies virus (PRV) infection promotes retrograde microtubule trafficking of the PRV capsid, also plays an important role in promoting nerve invasion and pathogenesis $[72,73]$. Microtubule trafficking of the viral capsid from the periphery of the cell to the nucleus is essential for viral replication. Viruses travel along the negative direction of microtubules toward the microtubule organization center near nucleus [66]. HSV-1 capsids colocalized with cytoplasmic dynein while in transit from the plasma membrane to the nucleus, so dose PRV and EBV [74-76]. HHV8 
modulates microtubule dynamics via RhoA-GTPdiaphanous 2 signaling and utilizes the dynein motors to deliver its DNA to the nucleus [59].

The stability and dynamics of microtubules are regulated by virus during the transport from the edge of the host cell to the nucleus. Soluble TAT or ectopic expression of HIV-1 TAT promoted KSHV oncogene Orf-K1 induced cell proliferation, as well as microtubule formation by targeting IKBa to activate the NF-kB pathway with the mediation of miR-891a-5p to induce abnormal angiogenesis, which is a main feature of aggressive AIDS-related Kaposi's sarcoma (AIDS-KS) [77]. Herpes simplex virus particles that have entered primary human cells exploit a dynamic specialized plus end-tracking proteins (+TIP) complex, comprised of end-binding protein 1 (EB1), cytoplasmic linker protein 170 (CLIP-170), and dynactin-1(DCTN1), to initiate retrograde transport [78]. The microtubule cytoskeleton undergoes a rapid and transient increase induced by EBV replication as well [79].

\section{The Interaction between Microtubules and Virus during Viral Egress}

The virus capsids reach the cell periphery along the plus-end of the microtubule. Microtubulemediated anterograde transport of herpes simplex virus (HSV) is crucial for the spread and transmission of the virus [80]. Kinesin-3 interacts with the viral membrane protein pUs9 during viral transport of the virus capsid in the early stages of infection with pseudorabies virus (PrV), and this interaction is attenuated when the virus reaches the neuronal axons [81]. KIF1A is a microtubule dependent kinesin-3 motor involved in axonal sorting and transport. Viral particles are co-transported with KIF1A in the axons of primary rat superior cervical ganglion neurons and overexpression or disruption of KIF1A function increases and reduces anterograde capsid transport, respectively. Us9 and KIF1A interact early during infection with the aid of additional viral protein(s) but they exhibit diminished binding at later stages when capsids typically stall in axons [81]. MT-binding protein dystonin (BPAG1) promotes trafficking of the virus from the centrosome to the cells along the positive end of the microtubule when there is a Herpes Simplex Virus 1 infection [82]. Dystonin (BPAG1) is also recruited by the capsid-binding protein pUL37 to the HSV-1 capsid to promote viral egress from the cytoplasm [83]. It has been shown that HDAC6 promotes assembly of IAV along microtubules to the plasma membrane by promoting microtubule acetylation [84]. HDAC6 in tumor cells appears to increase replication of oncolytic herpes simplex virus type 1 (oHSV) and alter the trafficking of post-entry oncolytic viral particles (OVs) from the nucleus to lysosomes [21]. The majority of HSV-containing organelles attached to the microtubules have a simple, minimal reconstitution of microtubule-mediated anterograde traffic that facilitates and complements molecular analysis of the HSV egress in vivo [80].

\section{The Interaction between Intermediate Filaments and Virus during Infection}

Egress from the nucleus requires herpesvirus capsids to traverse the inner nuclear membrane, an intermediate filaments meshwork composed of lamins and lamin-associated polypeptides [85]. MCMV egress from nuclear via its M50/p35 and M53/p38 proteins. M50 was inserted into the inner nuclear membrane (INM) and concentrated into distinct sites by M53. M50 then recruited cellular protein kinase C (PKC) [86-88], which phosphorylated lamins, thereby destabilizing the nuclear lamina layer [89]. The HSV-1 UL31 and UL34, M50 and M53 homologs in HSV and PRV, could bind lamin A/C in vitro, and caused lamin conformational changes in the nuclear [90-92]. PKC was also recruited to the nuclear rim depending on the presence of UL31 and UL34 [93]. Emerin, an INM protein that binds directly to lamin A/C, was also phosphorylated and delocalized during HSV infection, contributing to the egress of viral capsids from the nucleus [94].

\section{Cytoskeleton Components during the Late Stage of Infection}

In a latent infection, the virus has a very strong relationship with the cytoskeleton. Infection of most cells by herpesviruses causes a dramatic rearrangement of actin filaments and microtubules. HSV-infected cells showed a reorganization of actin filaments and a continuous decrease of F-actin [95]. Actin stress fibers dissociate by the viral Us3 serine/threonine kinase during alpha herpesvirus infection [96-99]. In consistent with actin filaments, the microtubule cytoskeleton undergoes a rapid and transient increase induced by EBV lysis and replication [100]. BGLF4, which is an EBV-encoded kinase that phosphorylates serine residues 25 and 38 of stathmin during mitosis, contributes to alterations in cellular MT dynamics during the EBV lytic cycle in EBV-harboring NPC [101]. The EBV-encoded protein LMP1 oncogene plays an important role in microtubules. LMP1 promotes microtubule degradation through ERK-mediated down-regulation of Op18/stathmin signaling in nasopharyngeal carcinoma cell [102]. LMP1 upregulates Cdc42 kinase activity, and oncoprotein 18/stathmin (Op18/ stathmin) phosphorylation promotes microtubule 
polymerization during mitosis, which leads to nasopharyngeal carcinogenesis [103]. LMP1 down-regulates the expression of RASSF1A, which is a protein that stabilizes microtubules depending on the activation of intracellular signaling of NF- $\mathrm{kB}$, and this downregulation may facilitate transformation of premalignant nasopharyngeal epithelial cells into cancer cells [104-107]. In addition, LMP1 interacts with FGD4 to activate Cdc42 and thereby leading to actin cytoskeleton rearrangement and increased motility of NPC cells [108-110]. EBV-encoded miRNAs [111] such as BART6-3p [112] and BART-10-3p [113] regulate microfilaments through downstream target genes.

\section{Cytoskeletal Drugs and Small-molecular Inhibitor on Viral Infection}

Intact cytoskeleton and cytoskeletal dynamic affect viral infection and viral DNA nuclear transport. Studies have shown that cytoskeletal drugs, including the microtubule- inhibiting drugs colchicine, nocodazole or paclitaxel, and the anti-actin filament drug cytochalasin $\mathrm{D}$, do not alter the redistribution, or the abundance of viral proteins or replication complexes increases or decreases, i.e., does not affect viral replication in the cells during viral replication [114]. However, Latrunculin A, which a F-actin-inhibiting drug, stalled nuclear capsids and induced actin rods to form in the nucleus. Immobile capsids accumulated around actin rods, and capsid motility stopped because latrunculin-induced actin rods nonspecifically bind nuclear capsids [61]. Viral particles are colocalized with microtubules and dynein proteins in vivo. The DNA trafficking was reduced by an inhibitor of the dynein motor and overexpression of dynamitin [115-123]. The co-localization of the microtubules and the viral capsid can also be disrupted by the microtubule drug nocodazole [59]. Nocodazole induces the expression of virus proteins, in addition to disrupting the co-localization of the microtubules and the viral capsid. Microtubule drugs affect the interaction of microtubules and viruses. Treatment with nocodazole, which provokes the depolymerization of microtubules, induces the expression of two EBV lytic cycle proteins, Zta and EA-D, which was mediated by transcriptional upregulation via protein kinase $C$ (PKC).In experiments with the microtubule destabilizing drug nocodazole, viral oncoprotein EBNA3C disrupted the mitotic spindle detection point [124]. Pre-treatment with inhibitors for PKC or its downstream signaling partners p38 mitogen-activated protein kinase (MAPK) and c-Jun $\mathrm{N}$-terminal kinase (JNK) abolished the nocodazole-mediated induction of two EBV lytic cycle proteins, Zta and EA-D in EBV-positive NPC cells [125]. DNA transporting of the virus can also be affected by other drugs. Deactivation of Rho GTPases

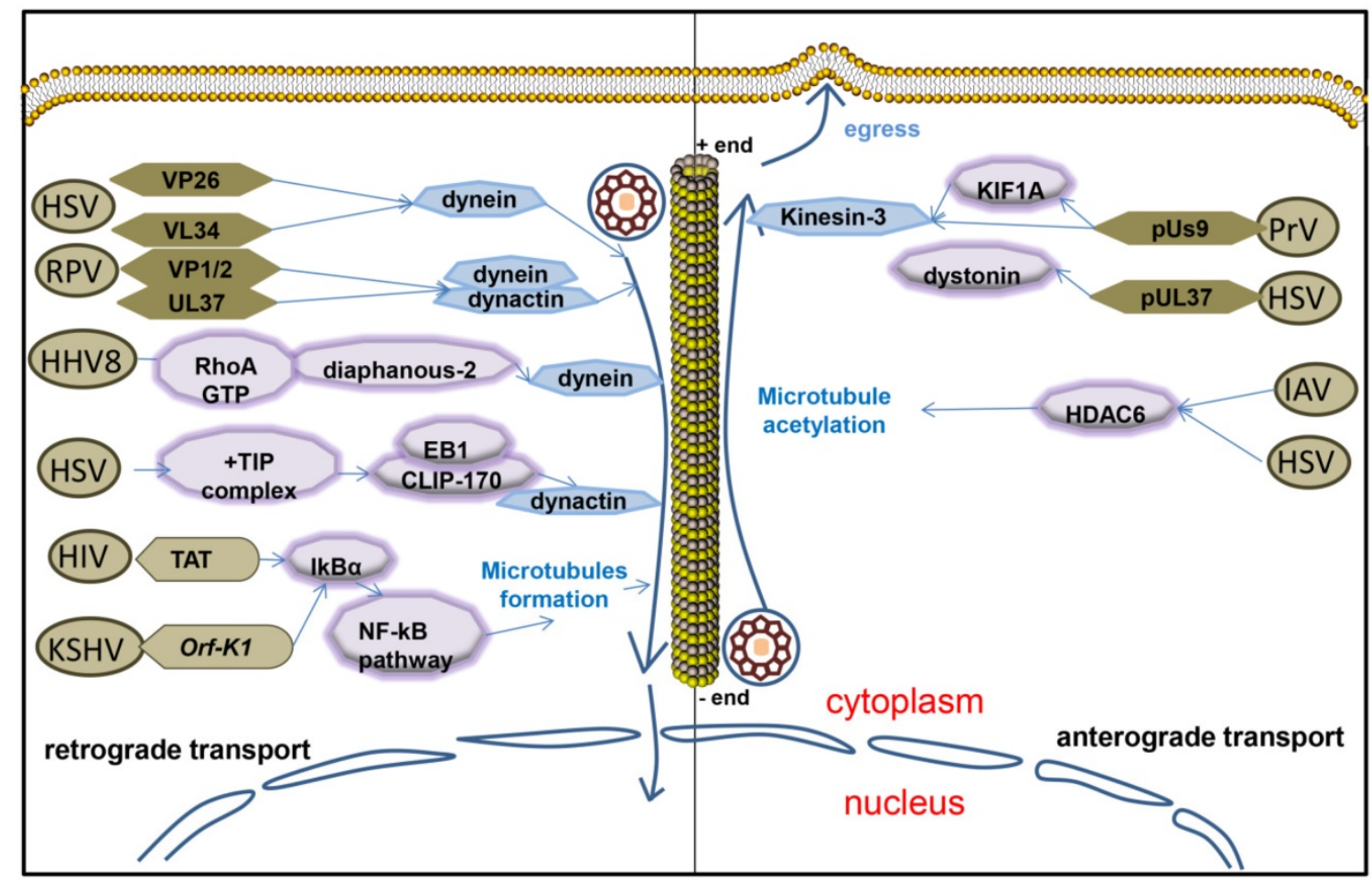

Figure 2. Microtubules interact with herpesvirus during viral infection. Herpesvirus retrograde transports along microtubule by interacting with dynein or dynein/dynactin complex when virus targets nucleus. After egresses from nucleus, herpesvirus anterograde transports along microtubule by interacting with kinesin or acetylating microtubule. 
by Clostridium difficile toxin B can significantly reduce the acetylation of microtubules and inhibit the transport of viral DNA to the nucleus, whereas activation of Rho GTPases by Escherichia coli cytotoxic necrotizing factor promotes the nuclear transport of viral DNA [59]. The microtubule inhibitor podofilox can preferentially target microtubule networks in the plasma membrane. Podofilox limits an early step of virus entry at the cell surface to drastically reduce infection by herpes simplex 1 , which indicates that microtubules appear to be participating in the post-binding step of virus entry including the pre- and post-penetration events [126]. Currently, there is a small-molecule that has been found to have a good effect on viruses. R10015 is a lead compound that blocks LIMK activity by binding to the ATP-binding pocket. R10015 specifically blocks viral DNA synthesis, nuclear migration, and virion release [127]. Scientists hope that this molecule will provide inspiration for further research on virus suppression.

\section{Conclusions}

Each components of the cytoskeleton plays an important role in the course of herpesvirus infection. Microfilaments interact with viral glycoproteins to mediate entry of the viruses when viruses contact the cells. Once viruses enter the cytoplasm, they travel along the minus end of the microtubule with the help of molecular motors, and then reach the microtubule organization center and nucleus at last. In the nucleus periphery, actin mediates the entry of viral DNA into the nucleus. After the replication of the viral DNA is completed, the viral DNA exits the nucleus under the action of the intermediate filaments and proceeds along the positive end of the microtubules toward the edges of the cells. Those cytoskeleton components are not independent of virus infection, but are coordinated with each other to participate in the virus infection. The virus capsid is released into the cytoplasm after fusion of the viral membrane and the host membrane, so it interacts with actin along microtubules to target the nuclear membrane [128]. During those processes, the cytoskeleton interacts with the virus, and the virus also changes the cytoskeleton, which affects the development of some virus-related tumors. However, there are still some questions that have not been answered. For example, how do the interactions between actin and microtubule, the interactions between cytoskeleton components and motor proteins, and the interactions between motor proteins and virus proteins facilitate trafficking within the cell? Atomic force microscopy (AFM) could be used as a tool for high resolution research into the ultrastructure and mechanical properties of tumor cells [129]. Moreover, the mechanism by which the virus affects the development of tumors through the cytoskeleton also requires further exploration.

\section{Acknowledgments}

This work was supported in part by grants from the National Natural Science Foundation of China (81572787, 81672683, 81672993, 81772928, 81702907 and 81772901) and the Natural Science Foundation of Hunan Province (2016JC2035, 2017SK2105, 2018JJ 3815, 2018JJ3704, 2018SK21210 and 2018SK21211).

\section{Competing Interests}

The authors have declared that no competing interest exists.

\section{References}

1. Roizman B, Carmichael LE, Deinhardt F, et al. Herpesviridae. Intervirology. 1981; 16: 201-17.

2. Dang L, Teng M, Li HZ, et al. Marek's disease virus type 1 encoded analog of miR-155 promotes proliferation of chicken embryo fibroblast and DF-1 cells by targeting hnRNPAB. Vet Microbiol. 2017; 207: 210-8.

3. $\mathrm{Li} \mathrm{L}, \mathrm{Gu}$ B, Zhou F, et al. Human herpesvirus $6 \mathrm{~A}$ infects human embryonic fibroblasts and induces G2/M arrest and cell death. J Med Virol. 2012; 84: 657-63.

4. Fan C, Tang $Y$, Wang J, et al. The emerging role of Epstein-Barr virus encoded microRNAs in nasopharyngeal carcinoma. J Cancer. 2018; 9: 2852-64.

5. Tu C, Zeng Z, Qi P, et al. Identification of genomic alterations in nasopharyngeal carcinoma and nasopharyngeal carcinoma-derived Epstein-Barr virus by whole-genome sequencing. Carcinogenesis. 2018; 39: 1517-28

6. Fan C, Wang J, Tang Y, et al. Long non-coding RNA LOC284454 promotes migration and invasion of nasopharyngeal carcinoma via modulating the Rho/ Rac signaling pathway. Carcinogenesis. 2019;40:380-91.

7. Tang L, Wei F, Wu Y, et al. Role of metabolism in cancer cell radioresistance and radiosensitization methods. J Exp Clin Cancer Res. 2018; 37: 87.

8. Tu C, Zeng Z, Qi P, et al. Genome-Wide Analysis of 18 Epstein-Barr Viruses Isolated from Primary Nasopharyngeal Carcinoma Biopsy Specimens. J Virol. 2017; 91: e00301-17.

9. Song $\mathrm{Y}$, Li X, Zeng Z, et al. Epstein-Barr virus encoded miR-BART11 promotes inflammation-induced carcinogenesis by targeting FOXP1. Oncotarget. 2016; 7:36783-99.

10. Xiao K, Yu Z, Li X, et al. Genome-wide Analysis of Epstein-Barr Virus (EBV) Integration and Strain in C666-1 and Raji Cells. J Cancer. 2016; 7: 214-24.

11. Wu C, Li M, Meng H, et al. Analysis of status and countermeasures of cancer incidence and mortality in China. Sci China Life Sci. 2019; 62:640-7

12. Wei $\mathrm{F}, \mathrm{Wu} \mathrm{Y}$, Tang $\mathrm{L}$, et al. Trend analysis of cancer incidence and mortality in China. Sci China Life Sci. 2017; 60: 1271-5.

13. Xiong $\mathrm{W}$, Zeng ZY, Xia JH, et al. A susceptibility locus at chromosome 3p21 linked to familial nasopharyngeal carcinoma. Cancer Res. 2004; 64: 1972-4.

14. Zeng Z, Zhou Y, Zhang W, et al. Family-based association analysis validates chromosome 3 p21 as a putative nasopharyngeal carcinoma susceptibility locus. Genet Med. 2006; 8: 156-60.

15. Zeng $Z$, Huang $H$, Zhang $W$, et al. Nasopharyngeal carcinoma: advances in genomics and molecular genetics. Sci China Life Sci. 2011; 54: 966-75.

16. Lin L, Lee JY, Kaplan LD, et al. Effects of chemotherapy in AIDS-associated non-Hodgkin's lymphoma on Kaposi's sarcoma herpesvirus DNA in blood. J Clin Oncol. 2009; 27: 2496-502.

17. Zhou F, Shimoda M, Olney L, et al. Oncolytic Reactivation of KSHV as a Therapeutic Approach for Primary Effusion Lymphoma. Mol Cancer Ther. 2017; 16: 2627-38.

18. Carbone A, De Paoli P, Gloghini A, et al. KSHV-associated multicentric Castleman disease: A tangle of different entities requiring multitarget treatment strategies. Int J Cancer. 2015; 137: 251-61.

19. Kumar B, Chandran B. KSHV Entry and Trafficking in Target Cells-Hijacking of Cell Signal Pathways, Actin and Membrane Dynamics. Viruses. 2016; 8: 305.

20. Wille PT, Wisner TW, Ryckman B, et al. Human cytomegalovirus (HCMV) glycoprotein $\mathrm{gB}$ promotes virus entry in trans acting as the viral fusion protein rather than as a receptor-binding protein. Mbio. 2013; 4: e00332-13.

21. Nakashima H, Kaufmann JK, Wang PY, et al. Histone deacetylase 6 inhibition enhances oncolytic viral replication in glioma. J Clin Invest. 2015; 125: 4269-80. 
22. Sodeik B, Ebersold MW, Helenius A. Microtubule-mediated transport of incoming herpes simplex virus 1 capsids to the nucleus. J Cell Biol. 1997; 136: 1007-21.

23. Zeng Z, Bo H, Gong Z, et al. AFAP1-AS1, a long noncoding RNA upregulated in lung cancer and promotes invasion and metastasis. Tumour Biol. 2016; 37: 729-37.

24. Bo H, Gong Z, Zhang W, et al. Upregulated long non-coding RNA AFAP1-AS1 expression is associated with progression and poor prognosis of nasopharyngeal carcinoma. Oncotarget. 2015; 6: 20404-18.

25. Wei F, Jing $\mathrm{YZ}, \mathrm{He} \mathrm{Y}$, et al. Cloning and characterization of the putative AFAP1-AS1 promoter region. J Cancer. 2019; 10: 1145-53.

26. Jayo A, Parsons M. Fascin: a key regulator of cytoskeletal dynamics. Int J Biochem Cell Biol. 2010; 42: 1614-7.

27. Feierbach B, Piccinotti S, Bisher M, et al. Alpha-Herpesvirus Infection Induces the Formation of Nuclear Actin Filaments. PLoS Pathog. 2006; 2: e85.

28. Forest T, Barnard S, Baines JD. Active intranuclear movement of herpesvirus capsids. Nat Cell Biol. 2005; 7: 429-31.

29. Xiong F, Deng S, Huang HB, et al. Effects and mechanisms of innate immune molecules on inhibiting nasopharyngeal carcinoma. Chin Med J (Engl). 2019; 132: 749-52.

30. Bo H, Fan L, Gong Z, et al. Upregulation and hypomethylation of lncRNA AFAP1AS1 predicts a poor prognosis and promotes the migration and invasion of cervical cancer. Oncol Rep. 2019; 41:2431-9.

31. Xia M, Zhang Y, Jin K, et al. Communication between mitochondria and other organelles: a brand-new perspective on mitochondria in cancer. Cell Biosci. 2019; 9: 27.

32. Howard J, Hyman AA. Dynamics and mechanics of the microtubule plus end. Nature. 2003; 422: 753-8.

33. Vale RD. The molecular motor toolbox for intracellular transport. Cell. 2003; 112: 467-80.

34. Pfister KK, Shah PR, Hummerich H, et al. Genetic Analysis of the Cytoplasmic Dynein Subunit Families. Plos Genetics. 2006; 2: e1.

35. Dohner K, Wolfstein A, Prank U, et al. Function of dynein and dynactin in herpes simplex virus capsid transport. Mol Biol Cell. 2002; 13: 2795-809.

36. Oriolo AS, Wald FA, Ramsauer VP, et al. Intermediate filaments: a role in epithelial polarity. Exp Cell Res. 2007; 313: 2255-64.

37. Zeng Z, Zhou Y, Xiong W, et al. Analysis of gene expression identifies candidate molecular markers in nasopharyngeal carcinoma using microdissection and cDNA microarray. J Cancer Res Clin Oncol. 2007; 133: 71-81.

38. Zeng ZY, Zhou $\mathrm{YH}$, Zhang WL, et al. Gene expression profiling of nasopharyngeal carcinoma reveals the abnormally regulated Wnt signaling pathway. Hum Pathol. 2007; 38: 120-33.

39. Zeng Z, Fan S, Zhang X, et al. Epstein-Barr virus-encoded small RNA 1 (EBER-1) could predict good prognosis in nasopharyngeal carcinoma. Clin Transl Oncol. 2016; 18: 206-11.

40. Shannon-Lowe C, Rowe M. Epstein Barr virus entry; kissing and conjugation. Curr Opin Virol. 2014; 4: 78-84

41. Lian Y, Xiong F, Yang L, et al. Long noncoding RNA AFAP1-AS1 acts as a competing endogenous RNA of miR-423-5p to facilitate nasopharyngeal carcinoma metastasis through regulating the Rho/Rac pathway. J Exp Clin Cancer Res. 2018; 37: 253

42. Tang Y, He Y, Zhang P, et al. LncRNAs regulate the cytoskeleton and related Rho/ROCK signaling in cancer metastasis. Mol Cancer. 2018; 17: 77.

43. Yang $\mathrm{L}$, Tang $\mathrm{Y}$, Xiong $\mathrm{F}$, et al. LncRNAs regulate cancer metastasis via binding to functional proteins. Oncotarget. 2017; 9: 1426-43.

44. Bo H, Fan L, Li J, et al. High Expression of lncRNA AFAP1-AS1 Promotes the Progression of Colon Cancer and Predicts Poor Prognosis. J Cancer. 2018; 9: 4677-83.

45. Hoppe S, Schelhaas M, Jaeger V, et al. Early herpes simplex virus type 1 infection is dependent on regulated Rac1/Cdc42 signalling in epithelial MDCKII cells. J Gen Virol. 2006; 87: 3483-94.

46. Wei F, Wu Y, Tang L, et al. BPIFB1 (LPLUNC1) inhibits migration and invasion of nasopharyngeal carcinoma by interacting with VTN and VIM. Br J Cancer. 2018; 118: 233-47.

47. Wei F, Tang L, He Y, et al. BPIFB1 (LPLUNC1) inhibits radioresistance in nasopharyngeal carcinoma by inhibiting VTN expression. Cell Death Dis. 2018; 9: 432.

48. He $\mathrm{Y}$, Jing $\mathrm{Y}$, Wei $\mathrm{F}$, et al. Long non-coding RNA PVT1 predicts poor prognosis and induces radioresistance by regulating DNA repair and cell apoptosis in nasopharyngeal carcinoma. Cell Death Dis. 2018; 9: 235.

49. Tang Y, Wang J, Lian Y, et al. Linking long non-coding RNAs and SWI/SNF complexes to chromatin remodeling in cancer. Mol Cancer. 2017; 16: 42

50. Goley ED, Ohkawa T, Mancuso J, et al. Dynamic nuclear actin assembly by Arp2/3 complex and a baculovirus WASP-like protein. Science. 2006; 314: 464-7.

51. Yoder A, Yu D, Dong L, et al. HIV envelope-CXCR4 signaling activates cofilin to overcome cortical actin restriction in resting CD4 T cells. Cell. 2008; 134: 782-92.

52. Tang Y, He Y, Shi L, et al. Co-expression of AFAP1-AS1 and PD-1 predicts poor prognosis in nasopharyngeal carcinoma. Oncotarget. 2017; 8: 39001-11.

53. Conti MA, Adelstein RS. Nonmuscle myosin II moves in new directions. J Cell Sci. $2008 ; 121: 11-8$
54. Vicentemanzanares $\mathrm{M}, \mathrm{Ma}$ X, Adelstein RS, et al. Non-muscle myosin II takes centre stage in cell adhesion and migration. Nat Rev Mol Cell Biol. 2009; 10: 778-90.

55. Wang M, Zhao J, Zhang L, et al. Role of tumor microenvironment in tumorigenesis. J Cancer. 2017; 8: 761-73.

56. Jiang $X$, Wang J, Deng $X$, et al. Role of the tumor microenvironment in PD-L1/PD-1-mediated tumor immune escape. Mol Cancer. 2019; 18: 10.

57. Duan S, Guo W, Xu Z, et al. Natural killer group 2D receptor and its ligands in cancer immune escape. Mol Cancer. 2019; 18: 29.

58. Wang JP, Tang YY, Fan CM, et al. The role of exosomal non-coding RNAs in cancer metastasis. Oncotarget. 2018; 9: 12487-502.

59. Naranatt PP, Krishnan HH, Smith MS, et al. Kaposi's sarcoma-associated herpesvirus modulates microtubule dynamics via RhoA-GTP-diaphanous 2 signaling and utilizes the dynein motors to deliver its DNA to the nucleus. J Virol. 2005; 79: 1191-206

60. Jacob T, Van den Broeke C, Van Waesberghe C, et al. Pseudorabies virus US3 triggers RhoA phosphorylation to reorganize the actin cytoskeleton. J Gen Virol. 2015; 96: 2328-35.

61. Bosse JB, Virding S, Thiberge SY, et al. Nuclear herpesvirus capsid motility is not dependent on F-actin. MBio. 2014; 5: e01909-14.

62. Wilkie AR, Lawler JL, Coen DM. A Role for Nuclear F-Actin Induction in Human Cytomegalovirus Nuclear Egress. MBio. 2016; 7: e01254-16.

63. Zou Z, Meng Z, Ma C, et al. Guanylate-Binding Protein 1 Inhibits Nuclear Delivery of Kaposi's Sarcoma-Associated Herpesvirus Virions by Disrupting Formation of Actin Filament. J Virol. 2017; 91: e00632-17.

64. Dohner K, Nagel CH, Sodeik B. Viral stop-and-go along microtubules: taking a ride with dynein and kinesins. Trends Microbiol. 2005; 13: 320-7.

65. Lyman MG, Enquist LW. Herpesvirus interactions with the host cytoskeleton. J Virol. 2009; 83: 2058-66.

66. Dammermann A, Desai A, Oegema K. The minus end in sight. Curr Biol. 2003; 13: R614-24

67. Damania B, Radtke K, Kieneke D, et al. Plus- and Minus-End Directed Microtubule Motors Bind Simultaneously to Herpes Simplex Virus Capsids Using Different Inner Tegument Structures. PLoS Pathogens. 2010; 6: e1000991.

68. Douglas MW, Diefenbach RJ, Homa FL, et al. Herpes simplex virus type 1 capsid protein VP26 interacts with dynein light chains RP3 and Tctex1 and plays a role in retrograde cellular transport. J Biol Chem. 2004; 279: 28522-30.

69. Reynolds AE, Wills EG, Roller RJ, et al. Ultrastructural localization of the herpes simplex virus type 1 UL31, UL34, and US3 proteins suggests specific roles in primary envelopment and egress of nucleocapsids. J Virol. 2002; 76: 8939-52.

70. Haverlock S, Coller KE, Antinone SE, et al. Targeting of Herpesvirus Capsid Transport in Axons Is Coupled to Association with Specific Sets of Tegument Proteins. Proc Natl Acad Sci U S A. 2005; 102: 5832-7.

71. Wolfstein A, Nagel CH, Radtke K, et al. The Inner Tegument Promotes Herpes Simplex Virus Capsid Motility Along Microtubules in vitro. Traffic. 2006; 7: 227-37.

72. Zaichick SV, Bohannon KP, Hughes A, et al. The herpesvirus VP1/2 protein is an effector of dynein-mediated capsid transport and neuroinvasion. Cell Host Microbe. 2013; 13: 193-203.

73. Liu YT, Jiang J, Bohannon KP, et al. A pUL25 dimer interfaces the pseudorabies virus capsid and tegument. J Gen Virol. 2017; 98: 2837-49.

74. Granzow H, Klupp BG, Mettenleiter TC. Entry of pseudorabies virus: an immunogold-labeling study. J Virol. 2005; 79: 3200-5.

75. Hutt-Fletcher LM. Epstein-Barr virus entry. J Virol. 2007; 81: 7825-32

76. Zou G, Ren B, Liu Y, et al. Inhibin B suppresses anoikis resistance and migration through the transforming growth factor-beta signaling pathway in nasopharyngeal carcinoma. Cancer Sci. 2018; 109: 3416-27.

77. Yao S, Hu M, Hao T, et al. MiRNA-891a-5p mediates HIV-1 Tat and KSHV Orf-K1 synergistic induction of angiogenesis by activating NF-kappaB signaling. Nucleic Acids Res. 2015; 43: 9362-78.

78. Jovasevic V, Naghavi MH, Walsh D. Microtubule plus end-associated CLIP-170 initiates HSV-1 retrograde transport in primary human cells. J Cell Biol. 2015; 211: 323-37.

79. Zhou Y, Liao Q, Li X, et al. HYOU1, Regulated by LPLUNC1, Is Up-Regulated in Nasopharyngeal Carcinoma and Associated with Poor Prognosis. J Cancer. 2016; 7: 367-76

80. Lee GE, Murray JW, Wolkoff AW, et al. Reconstitution of herpes simplex virus microtubule-dependent trafficking in vitro. J Virol. 2006; 80: 4264-75.

81. Kramer T, Greco TM, Taylor MP, et al. Kinesin-3 mediates axonal sorting and directional transport of alphaherpesvirus particles in neurons. Cell Host Microbe. 2012; 12: 806-14

82. McElwee M, Beilstein F, Labetoulle M, et al. Dystonin/BPAG1 promotes plus-end-directed transport of herpes simplex virus 1 capsids on microtubules during entry. J Virol. 2013; 87: 11008-18.

83. Pasdeloup D, McElwee M, Beilstein F, et al. Herpesvirus tegument protein pUL37 interacts with dystonin/BPAG1 to promote capsid transport on microtubules during egress. J Virol. 2013; 87: 2857-67.

84. Husain M, Cheung CY. Histone deacetylase 6 inhibits influenza A virus release by downregulating the trafficking of viral components to the plasma membrane via its substrate, acetylated microtubules. J Virol. 2014; 88: 11229-39.

85. Lusk CP, Blobel G, King MC. Highway to the inner nuclear membrane: rules for the road. Nat Rev Mol Cell Biol. 2007; 8: 414-20. 
86. Zhang Y, Xia M, Jin K, et al. Function of the c-Met receptor tyrosine kinase in carcinogenesis and associated therapeutic opportunities. Mol Cancer. 2018; 17: 45.

87. Wang YA, Li XL, Mo YZ, et al. Effects of tumor metabolic microenvironment on regulatory T cells. Mol Cancer. 2018; 17: 168

88. Zhong Y, Du Y, Yang X, et al. Circular RNAs function as ceRNAs to regulate and control human cancer progression. Mol Cancer. 2018; 17: 79.

89. Muranyi W, Haas J, Wagner M, et al. Cytomegalovirus recruitment of cellular kinases to dissolve the nuclear lamina. Science. 2002; 297: 854-7.

90. Bjerke SL, Roller RJ. Roles for herpes simplex virus type 1 UL34 and US3 proteins in disrupting the nuclear lamina during herpes simplex virus type 1 egress. Virology. 2006; 347: 261-76.

91. Reynolds AE, Liang L, Baines JD. Conformational changes in the nuclear lamina induced by herpes simplex virus type 1 require genes $U(L) 31$ and U(L)34. J Virol. 2004; 78: 5564-75.

92. Xu K, Xiong $\mathrm{W}$, Zhou $\mathrm{M}$, et al. Integrating ChIP-sequencing and digital gene expression profiling to identify BRD7 downstream genes and construct their regulating network. Mol Cell Biochem. 2016; 411: 57-71.

93. Park R, Baines JD. Herpes simplex virus type 1 infection induces activation and recruitment of protein kinase $C$ to the nuclear membrane and increased phosphorylation of lamin B. J Virol. 2006; 80: 494-504.

94. Morris JB, Hofemeister H, O'Hare P. Herpes simplex virus infection induces phosphorylation and delocalization of emerin, a key inner nuclear membrane protein. J Virol. 2007; 81: 4429-37.

95. Bedows E, Rao KM, Welsh MJ. Fate of microfilaments in vero cells infected with measles virus and herpes simplex virus type 1. Mol Cell Biol. 1983; 3: 712-9.

96. Liang F, Li Q, Li X, et al. TSC22D2 interacts with PKM2 and inhibits cell growth in colorectal cancer. Int J Oncol. 2016; 49: 1046-56.

97. Li Q, Chen P, Zeng Z, et al. Yeast two-hybrid screening identified WDR77 as a novel interacting partner of TSC22D2. Tumour Biol. 2016; 37: 12503-12.

98. Yu J, Liu Y, Gong Z, et al. Overexpression long non-coding RNA LINC00673 is associated with poor prognosis and promotes invasion and metastasis in tongue squamous cell carcinoma. Oncotarget. 2017; 8: 16621-32.

99. Yu J, Liu Y, Guo C, et al. Upregulated long non-coding RNA LINC00152 expression is associated with progression and poor prognosis of tongue squamous cell carcinoma. J Cancer. 2017; 8: 523-30.

100. LaJeunesse DR, Brooks K, Adamson AL. Epstein-Barr virus immediate-early proteins BZLF1 and BRLF1 alter mitochondrial morphology during lytic replication. Biochem Biophys Res Commun. 2005; 333: 438-42.

101. Chen PW, Lin SJ, Tsai SC, et al. Regulation of microtubule dynamics through phosphorylation on stathmin by Epstein-Barr virus kinase BGLF4. J Biol Chem. 2010; 285: 10053-63.

102. Lin X, Tang M, Tao $\mathrm{Y}$, et al. Epstein-Barr virus-encoded LMP1 triggers regulation of the ERK-mediated Op18/stathmin signaling pathway in association with cell cycle. Cancer Sci. 2012; 103: 993-9.

103. Lin X, Liu S, Luo X, et al. EBV-encoded LMP1 regulates Op18/stathmin signaling pathway by cdc 2 mediation in nasopharyngeal carcinoma cells. Int J Cancer. 2009; 124: 1020-7.

104. Man C, Rosa J, Lee LT, et al. Latent membrane protein 1 suppresses RASSF1A expression, disrupts microtubule structures and induces chromosomal aberrations in human epithelial cells. Oncogene. 2007; 26: 3069-80.

105. Yang L, Tang Y, He Y, et al. High Expression of LINC01420 indicates an unfavorable prognosis and modulates cell migration and invasion in nasopharyngeal carcinoma. J Cancer. 2017; 8: 97-103.

106. Zhang W, Fan S, Zou G, et al. Lactotransferrin could be a novel independent molecular prognosticator of nasopharyngeal carcinoma. Tumour Biol. 2015; 36: $675-83$

107. Zhang W, Zeng Z, Wei F, et al. SPLUNC1 is associated with nasopharyngeal carcinoma prognosis and plays an important role in all-trans-retinoic acid-induced growth inhibition and differentiation in nasopharyngeal cancer cells. FEBS J. 2014; 281: 4815-29.

108. Fan CM, Wang JP, Tang YY, et al. circMAN1A2 may serve as a novel serum biomarker for malignant tumors. Cancer Sci. 2019. doi: 10.1111/cas.14034. [Epub ahead of print].

109. Zhang W, Zeng Z, Fan S, et al. Evaluation of the prognostic value of TGF-beta superfamily type I receptor and TGF-beta type II receptor expression in nasopharyngeal carcinoma using high-throughput tissue microarrays. J Mol Histol. 2012; 43: 297-306.

110. Zhang W, Huang C, Gong Z, et al. Expression of LINC00312, a long intergenic non-coding RNA, is negatively correlated with tumor size but positively correlated with lymph node metastasis in nasopharyngeal carcinoma. J Mol Histol. 2013; 44: 545-54.

111. Zeng Z, Huang H, Huang L, et al. Regulation network and expression profiles of Epstein-Barr virus-encoded microRNAs and their potential target host genes in nasopharyngeal carcinomas. Sci China Life Sci. 2014; 57: 315-26.

112. He B, Li W, Wu Y, et al. Epstein-Barr virus-encoded miR-BART6-3p inhibits cancer cell metastasis and invasion by targeting long non-coding RNA LOC553103. Cell Death Dis. 2016; 7: e2353.

113. Yan Q, Zeng Z, Gong Z, et al. EBV-miR-BART10-3p facilitates epithelial-mesenchymal transition and promotes metastasis of nasopharyngeal carcinoma by targeting BTRC. Oncotarget. 2015; 6: 41766-82.

114. Greber UF, Way M. A superhighway to virus infection. Cell. 2006; 124: 741-54.
115. Zhang W, Greene W, Gao SJ. Microtubule- and dynein-dependent nuclear trafficking of rhesus rhadinovirus in rhesus fibroblasts. J Virol. 2012; 86: 599-604.

116. Fan $\mathrm{C}$, Tang $\mathrm{Y}$, Wang $\mathrm{J}$, et al. Role of long non-coding RNAs in glucose metabolism in cancer. Mol Cancer. 2017; 16: 130.

117. Zhou R, Wu Y, Wang W, et al. Circular RNAs (circRNAs) in cancer. Cancer Lett. 2018; 425: 134-42.

118. He R, Liu P, Xie X, et al. circGFRA1 and GFRA1 act as ceRNAs in triple negative breast cancer by regulating miR-34a. J Exp Clin Cancer Res. 2017; 36: 145.

119. Wang Y, Mo Y, Gong Z, et al. Circular RNAs in human cancer. Mol Cancer. 2017; 16: 25

120. Wang Y, Mo Y, Yang X, et al. Long non-coding RNA AFAP1-AS1 is a novel biomarker in various cancers: a systematic review and meta-analysis based on the literature and GEO datasets. Oncotarget. 2017; 8: 102346-60.

121. Wang Y, Xue D, Li Y, et al. The Long Noncoding RNA MALAT-1 is A Novel Biomarker in Various Cancers: A Meta-analysis Based on the GEO Database and Literature. J Cancer. 2016; 7: 991-1001.

122. Gong Z, Yang Q, Zeng Z, et al. An integrative transcriptomic analysis reveals p53 regulated miRNA, mRNA, and lncRNA networks in nasopharyngeal carcinoma. Tumour Biol. 2016; 37: 3683-95.

123. Gong Z, Zhang S, Zeng Z, et al. LOC401317, a p53-regulated long non-coding RNA, inhibits cell proliferation and induces apoptosis in the nasopharyngeal carcinoma cell line HNE2. PLoS One. 2014; 9: e110674.

124. Parker GA, Touitou R, Allday MJ. Epstein-Barr virus EBNA3C can disrupt multiple cell cycle checkpoints and induce nuclear division divorced from cytokinesis. Oncogene. 2000; 19: 700-9.

125. Liu YR, Huang SY, Chen JY, et al. Microtubule depolymerization activates the Epstein-Barr virus lytic cycle through protein kinase $C$ pathways in nasopharyngeal carcinoma cells. J Gen Virol. 2013; 94: 2750-8.

126. Cohen T, Schwarz T, Vigant F, et al. The Microtubule Inhibitor Podofilox Inhibits an Early Entry Step of Human Cytomegalovirus. Viruses. 2016; 8: 295.

127. Yi F, Guo J, Dabbagh D, et al. Discovery of Novel Small-Molecule Inhibitors of LIM Domain Kinase for Inhibiting HIV-1. J Virol. 2017; 91: e02418-16.

128. Huffman JB, Daniel GR, Falck-Pedersen E, et al. The C Terminus of the Herpes Simplex Virus UL25 Protein Is Required for Release of Viral Genomes from Capsids Bound to Nuclear Pores. J Virol. 2017; 91: e00641-17.

129. Deng X, Xiong F, Li X, et al. Application of atomic force microscopy in cancer research. J Nanobiotechnology. 2018; 16: 102. 